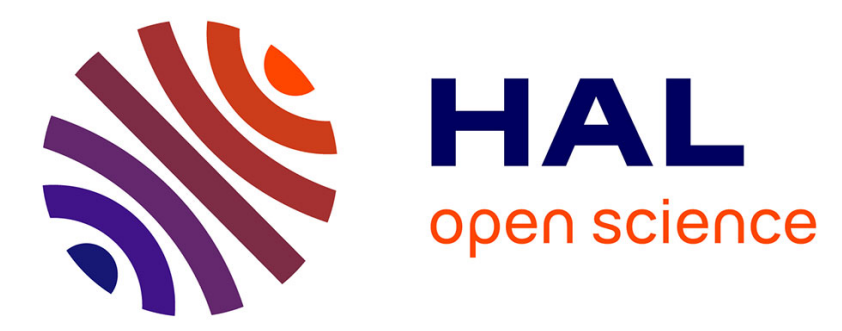

\title{
Highly tunable sulfur hexafluoride separation by interpenetration control in metal organic frameworks
}

Ioannis Skarmoutsos, Mohamed Eddaoudi, Guillaume Maurin

\section{To cite this version:}

Ioannis Skarmoutsos, Mohamed Eddaoudi, Guillaume Maurin. Highly tunable sulfur hexafluoride separation by interpenetration control in metal organic frameworks. Microporous and Mesoporous Materials, 2019, 281, pp.44-49. 10.1016/j.micromeso.2019.02.035 . hal-02166273

\section{HAL Id: hal-02166273 \\ https://hal.umontpellier.fr/hal-02166273}

Submitted on 22 Oct 2021

HAL is a multi-disciplinary open access archive for the deposit and dissemination of scientific research documents, whether they are published or not. The documents may come from teaching and research institutions in France or abroad, or from public or private research centers.
L'archive ouverte pluridisciplinaire HAL, est destinée au dépôt et à la diffusion de documents scientifiques de niveau recherche, publiés ou non, émanant des établissements d'enseignement et de recherche français ou étrangers, des laboratoires publics ou privés.

\section{다)(1) $(5$}

Distributed under a Creative Commons Attribution - NonCommerciall 4.0 International 


\title{
Highly tunable sulfur hexafluoride separation by interpenetration control in Metal Organic Frameworks
}

\author{
Ioannis Skarmoutsos ${ }^{\mathrm{a}^{*}}$, Mohamed Eddaoudi ${ }^{\mathrm{b}}$, Guillaume Maurin ${ }^{\mathrm{a}}$ \\ anstitut Charles Gerhardt Montpellier, UMR 5253 CNRS, Université de Montpellier, Place E. \\ Bataillon, 34095 Montpellier Cedex 05, France \\ *Corresponding Author: iskarmoutsos@ hotmail.com \\ bAdvanced Membranes and Porous Materials Center Division of Physical Sciences and Engineering, \\ King Abdullah University of Science and Technology (KAUST), P.O. Box 4700, Thuwal 23955-6900, \\ Kingdom of Saudi Arabia
}

\begin{abstract}
The separation of fluid $\mathrm{SF}_{6} / \mathrm{N}_{2}$ mixtures using the recently synthesized metal-organic framework SIFSIX-2-Cu and its interpenetrated polymorph SIFSIX-2-Cu-i has been systematically studied by employing Monte Carlo and Molecular Dynamics simulations. These predictions revealed that SIFSIX-2-Cu shows a high thermodynamic adsorption selectivity for $\mathrm{SF}_{6}$ over $\mathrm{N}_{2}$ combined with one of the highest $\mathrm{SF}_{6}$ uptakes reported so far for an adsorbent. On the other hand, the smaller pore dimensions of the interpenetrated network cause a molecular sieving effect, allowing only the adsorption of $\mathrm{N}_{2}$. Furthermore, the self-diffusivity of the adsorbed $\mathrm{SF}_{6}$ molecules in the SIFSIX-2-Cu framework was predicted to be significantly lower than the one corresponding to $\mathrm{N}_{2}$, while the smaller pore channel network of SIFSIX2-Cu-i decreases the self-diffusivity of $\mathrm{N}_{2}$ by a factor of about 6 . This study revealed that controlling the interpenetration in MOFs is an efficient way to tune their separation performances for strategic gas mixtures.
\end{abstract}




\section{Graphical Abstract}

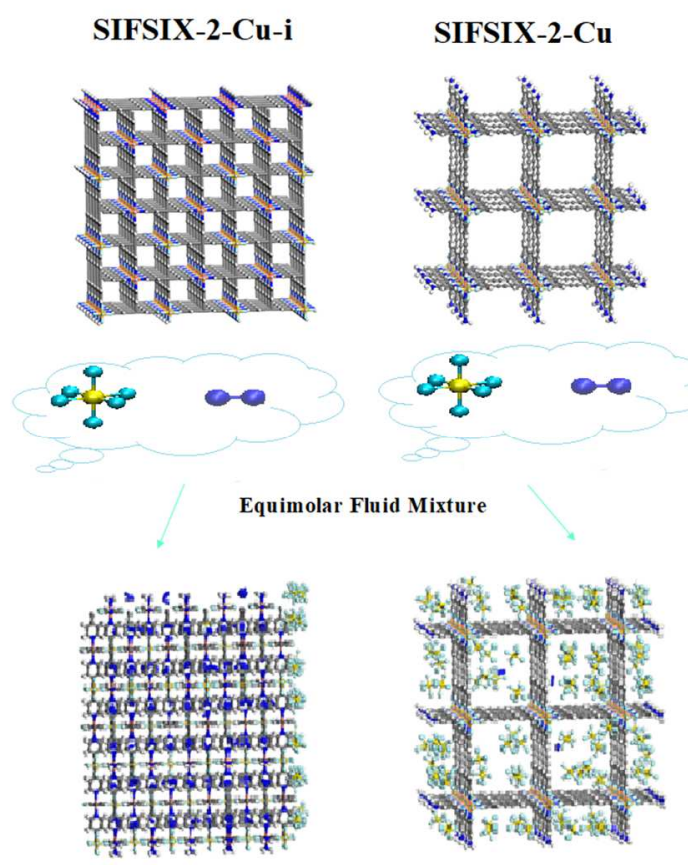

Keywords: sulfur hexafluoride, nitrogen, gas separation, metal-organic frameworks, framework interpenetration, SIFSIX-2-Cu-i, SIFSIX-2-Cu, Monte Carlo simulations, Molecular Dynamics simulations.

\section{Highlights}

- The separation of $\mathrm{SF}_{6} / \mathrm{N}_{2}$ fluid mixtures has been studied.

- The SIFSIX-2-Cu and its interpenetrated polymorph SIFSIX-2-Cu-i have been systematically examined.

- Force field-based Monte Carlo and molecular dynamics simulation techniques have been employed.

- A high thermodynamic adsorption selectivity for $\mathrm{SF}_{6}$ over $\mathrm{N}_{2}$ in the case of SIFSIX-2-Cu has been revealed.

- The smaller pore dimensions of the interpenetrated SIFSIX-2-Cu-i network cause a molecular sieving effect, allowing only the adsorption of $\mathrm{N}_{2}$. 


\section{Introduction}

Owing to its high dielectric strength and arc-quenching properties, low toxicity and high stability, sulfur hexafluoride $\left(\mathrm{SF}_{6}\right)$ is the most widely used insulating and switching medium for electrical power transmission equipment and several other applications, especially under high voltage [1-6]. However, $\mathrm{SF}_{6}$ is a very potent greenhouse gas due to its very long atmospheric lifetime [2] (3200 years) and its high efficiency as an infrared absorber [6]. This gas shows one of the highest global warming potentials, 24000 times higher than $\mathrm{CO}_{2}$ and it is amongst the main anthropogenic greenhouse gases [7]. In this context, the Kyoto protocol aims to reduce its emission [7]. Furthermore, $\mathrm{SF}_{6}$ falls also under the European F-Gas regulation limiting its use in diverse fields [5]. Therefore, there is a critical need to reduce the use of $\mathrm{SF}_{6}$ as an insulator in high voltage and circuit breakers. The current alternative consists of considering a pressurized gas mixture, where a low content of $\mathrm{SF}_{6}$ is mixed with ordinary gases such as $\mathrm{N}_{2}, \mathrm{CO}_{2}$ or air [8]. It was demonstrated that when mixing $\mathrm{SF}_{6}$ with $\mathrm{N}_{2}$, the fluid mixture maintains the high dielectric strength of pure $\mathrm{SF}_{6}$, even at high $\mathrm{N}_{2}$ concentration [9]. However, when mixing these two fluids the complexity of recovering and recycling $\mathrm{SF}_{6}$ increases [4] and this holds even more true when the amount of $\mathrm{SF}_{6}$ in the mixture decreases [9]. In this case, the development of efficient processes for the separation of $\mathrm{SF}_{6} / \mathrm{N}_{2}$ mixtures is required.

Adsorption [4-6,10-17] and membrane [8,18-21] based-separation have been recently proposed as low energy and cost alternative recovery methods to liquefaction using refrigeration [13]. The efficiency of such processes strongly relies on the choice of the optimal adsorbents combining fast permeability and high thermodynamic selectivity for the gas of interest. Several porous materials, such as zeolites, metal-organic frameworks (MOFs), carbon nanopores and porous organic cages have been envisaged for the separation of $\mathrm{SF}_{6} / \mathrm{N}_{2}$ mixtures. The most selective materials reported so far are zeolites FAU-ZTC [4] and 13X [10], a series of isostructural MMOF-74 materials with a high density of unsaturated metal sites [11] and the CC3 porous organic cage [15]. The $\mathrm{SF}_{6} / \mathrm{N}_{2}$ separation ability of these materials was attributed to a combination of fluid/pore interactions and pore dimensions, as well as to the flexible nature of the molecular cage crystal. 
More specifically, MOFs appear as a promising family of custom-designed adsorbents able to efficient capture strategic gases of utmost importance in diverse energy and environment-related applications [22,23]. The large variety of metal ions and organic ligands presents tremendous opportunities to design novel and fascinating entangled architectures, toward task-specific applications. MOFs showing interpenetrated networks correspond to the sub-class of MOFs that have been by far much less investigated for their potential uses in diverse applications [24,25]. Indeed, the structural interpenetration, closely associated with the pore shape/dimension of the adsorbent is expected to play a predominant role in the molecular separation process by favoring (i) a thermodynamically-driven capture via a subtle control of the degree of confinement and hence of the strength of the host/guest interactions, and/or (ii) a size exclusion via a strict control of the pore size to selectively adsorb only the smaller molecule of the mixture.

In this context, Eddaoudi et al reported recently a crystal engineering strategy controlling the pore functionality and size in a series of MOFs, by pillaring twodimensional nets of organic ligands and metal nodes with hexafluorosilicate $\left(\mathrm{SiF}_{6}{ }^{2-}\right)$ anions to form three-dimensional networks with primitive cubic (pcu) topology [26]. The pore size of this family of fluorinated MOFs, resembling pillared square grids, was tuned by either changing the length of the organic linker and/or the metal node, or using the framework interpenetration approach. In this study, $\left[\mathrm{Cu}\left(4,4^{\prime}-\right.\right.$ dipyridylacetylene $\left.)_{2}\left(\mathrm{SiF}_{6}\right)\right]_{\mathrm{n}}$, named as SIFSIX-2-Cu, was reported as a onedimensional square channel-like MOF with a pore dimension of $13.05 \AA$. Interestingly an interpenetrated polymorph isostructural to SIFSIX-2-Cu, named as SIFSIX-2-Cu-i, composed of double interpenetrated nets was also discovered. The independent nets are staggered with respect to one another, significantly reducing the dimension of the one-dimensional square pore channels to $5.15 \AA$ A. SIFSIX-2-Cu-i was demonstrated earlier [26] to remain in its pristine interpenetrated form after evacuation of solvent. This material, with a permanent porosity associated with an apparent BET area of $735 \mathrm{~m}^{2} / \mathrm{g}$, as well as all this family of fluorinated MOFs was shown to be an ideal platform for the selective capture of $\mathrm{CO}_{2}$ over a wide range of mixtures including $\mathrm{CO}_{2} / \mathrm{CH}_{4}, \mathrm{CO}_{2} / \mathrm{N}_{2}$ and $\mathrm{CO}_{2} / \mathrm{H}_{2}$ [26,27]. These findings motivated us to perform a computational exploration of the efficiency of this SIFSIX series of materials for the separation of the $\mathrm{SF}_{6} / \mathrm{N}_{2}$ mixtures at ambient conditions, particularly 
focusing on the impact of the interpenetrated network. Note that although only a few MOFs have been tested so far for the separation of $\mathrm{SF}_{6} / \mathrm{N}_{2}$ mixtures [4-6,10-17], some of them outperform other adsorbents such as zeolites and carbon-based materials [6]. Indeed some studies suggested that MOFs with small pore sizes might be adequate for trapping $\mathrm{SF}_{6}$ at low concentrations whereas MOFs with larger pore sizes will be adequate for the storage of this molecule [6].

\section{Computational Methods}

A combination of force field-based Grand Canonical Monte Carlo (GCMC) and molecular dynamics (MD) simulations was employed in order to explore the adsorption of $\mathrm{SF}_{6}$ and $\mathrm{N}_{2}$ as single fluids in the SIFSIX-2-Cu and SIFSIX-2-Cu-i MOFs and the separation of the $\mathrm{SF}_{6} / \mathrm{N}_{2}$ binary mixtures (two bulk molar compositions $\mathrm{SF}_{6} / \mathrm{N}_{2}: 1: 1$ and 1:9) at $303 \mathrm{~K}$, and in the pressure range 0.1-10 bar. Well-established all-atom rigid potential models for $\mathrm{SF}_{6}$ [28] and $\mathrm{N}_{2}$ [29] have been selected. The microscopic models for the MOFs, including the force field parameters, were defined following the same strategy than in our previous works [27,30]. More specifically, the CHELPG atomic charges were extracted from cluster-based density functional theory (DFT) calculations [27] while the sigma and epsilon 12-6 LennardJones (LJ) parameters were taken from the literature, the LJ parameters from the DREIDING [31] and the UFF [32] force fields being assigned to the atoms of the organic and inorganic building blocks of the MOF, respectively (see Ref. 27 for more details). Lorentz-Berthelot combining rules were employed for the LJ interactions between the different atoms of the MOF/guest pairs. The SIFSIX-2-Cu-i supercell used in our simulation studies has been taken from our previous work [27] and comprised $3 \times 3 \times 5$ unit cells. The SIFSIX-2-Cu supercell constituted by a $3 \times 3 \times 5$ replica of its unit cell, which was initially geometry optimized at the periodic DFT level. The employed density functional was the PBE [33] with the D3 dispersion correction introduced by Grimme [34]. A hybrid Gaussian and plane waves approach, as implemented in the Quickstep module [35] of the cp2k code [36] was employed, using the DZVP-MOLOPT basis set [37] and the Goedecker-Teter-Hutter pseudopotentials [38] for the electronic structure calculations. The density cut-off was set to $500 \mathrm{Ry}$ to yield well converged calculations. The supercell was saturated by adding terminal $-\mathrm{NH}_{2}$ groups and the charges of the terminal atoms were adjusted in order to give a total zero charge to the supercell. The names of the different 
interaction sites corresponding to a model building block of SIFSIX-2-Cu are presented in Figure 1. The force field parameters are also provided in Tables 1-3.

The GCMC simulations were carried out with the Complex Adsorption and Diffusion Simulation Suite (CADSS) code [39]. The fugacities for each adsorbed species at the investigated thermodynamic conditions were calculated using the Peng-Robinson equation of state [40]. For each simulated state point, $2 \times 10^{8}$ Monte Carlo steps were used for the equilibration and production runs. Several types of trial molecular moves were taken into account: translation or rotation, creation or deletion and exchange of molecular identity. MD simulations were further performed in the canonical NVT ensemble to explore the diffusivity of the guest molecules in both SIFSIX-2-Cu and SIFSIX-2-Cu-i. The equations of motion were integrated using a leapfrog-type Verlet algorithm [14] and the integration time step was set to $1 \mathrm{fs}$. The intramolecular geometry of $\mathrm{SF}_{6}$ and $\mathrm{N}_{2}$ molecules was constrained using the quaternion formalism [41]. A Nose-Hoover thermostat [42] with a temperature relaxation time of 0.2 ps was used to constrain the temperature during the simulations. All MD simulations were run for $20 \mathrm{~ns}$, after a $10 \mathrm{~ns}$ equilibration period, using the DL_POLY simulation code [43].

\section{Results and Discussion}

The simulated isotherms of $\mathrm{SF}_{6}$ and $\mathrm{N}_{2}$ as single components are first reported for both SIFSIX materials in Figure 2a. These data evidence that SIFSIX-2-Cu-i only adsorbs $\mathrm{N}_{2}$ since the pore size of this interpenetrated framework is small enough to exclude the adsorption of the bulkiest $\mathrm{SF}_{6}$ molecule. Further, the adsorption profile for SIFSIX-2-Cu suggests that this MOF shows a much higher affinity for $\mathrm{SF}_{6}$ vs $\mathrm{N}_{2}$ leading to only a negligible amount of $\mathrm{N}_{2}$ adsorbed in its pores. This trend was further supported by the calculated adsorption enthalpies at low coverage, which are -22.1 $\mathrm{kJ} / \mathrm{mol}$ and $-9.1 \mathrm{~kJ} / \mathrm{mol}$ for $\mathrm{SF}_{6}$ and $\mathrm{N}_{2}$ respectively. As a further step, the coadsorption isotherms were simulated for two $\mathrm{SF}_{6} / \mathrm{N}_{2}$ molar mixtures (1:1 and 1:9) in both SIFSIX materials as reported Figures $2 b-c$, respectively. Note that in all cases the pressure in the $\mathrm{x}$-axis is the total pressure of the system. These results confirmed the higher affinity for $\mathrm{SF}_{6}$ adsorption in SIFSIX-2-Cu. This behavior is again supported by a much higher adsorption enthalpy at low coverage for $\mathrm{SF}_{6}(-21.4 \mathrm{~kJ} / \mathrm{mol}, 1: 1 \mathrm{bulk}$ molar mixture) vs $\mathrm{N}_{2}(-9.2 \mathrm{~kJ} / \mathrm{mol}, 1: 1$ bulk molar mixture) that supports a 
thermodynamic-driven separation mechanism. A high $\mathrm{SF}_{6} / \mathrm{N}_{2}$ selectivity was simulated in the whole range of pressures (Figure 3), with a maximum of about 43 (bulk molar mixture 1:1) and 41 (bulk molar mixture 1:9) at pressures of 1.5 bar and 7 bar respectively. Again, in this case, as in Figure 2, the pressure in the $\mathrm{x}$-axis is the total pressure of the mixture. The presence of this maximum for both mixture compositions can be interpreted as a result of a competitive adsorption at high pressures, where due to packing effects the adsorption of $\mathrm{N}_{2}$ in the pore becomes easier, whereas at lower pressures the thermodynamic-driven separation mechanism is the predominant one. The shift of the selectivity maximum at higher pressures in the case of the 1:9 bulk molar mixture can be explained in terms of the lower bulk composition of $\mathrm{SF}_{6}$, leading to a lower uptake of $\mathrm{SF}_{6}$ and a higher uptake of $\mathrm{N}_{2}$, as observed in Figure 2 c. Due to the lower $\mathrm{SF}_{6}$ uptake, packing effects are more important at higher pressures, leading therefore to this shift of the selectivity maximum.

Note that for the 1:9 molar composition, the selectivity obtained for SIFSIX-2-Cu at 1 bar ( 25) is similar than the values reported for the Mg-, Co- and Zn-MOF-74 at 308 $\mathrm{K}$ [11] ( 20, 30 and 40, respectively). The selectivity for the 1:1 composition at ambient pressure is also comparable to the previously reported ones for $\mathrm{Mg}$ - and CoMOF-74 [11] ( 30 and 60, respectively) and the EMT-ZTC zeolite ( 60) [4], while still remaining lower than that reported for other porous solids including the CC3 porous organic cage [15] ( 200) and the FAU-ZTC zeolite [4] ( 130). However, interestingly SIFSIX-2-Cu shows a much larger $\mathrm{SF}_{6}$ uptake as compared with other materials $[6,11]$, while the relatively moderate $\mathrm{SF}_{6}$ adsorption enthalpy suggests a rather easy regeneration. A very recent publication [44] lists the most efficient adsorbents in terms of $\mathrm{SF}_{6}$ uptake and $\mathrm{SF}_{6} / \mathrm{N}_{2}$ selectivity. It comes that SIFSIX-2-Cu is one the most efficient adsorbents reported so the capture of $\mathrm{SF}_{6}$ combining an extremely large $\mathrm{SF}_{6}$ uptake and a high $\mathrm{SF}_{6} / \mathrm{N}_{2}$ selectivity. All together, these features make this material as a promising candidate to selectively adsorb $\mathrm{SF}_{6}$ from $\mathrm{N}_{2}$ via a thermodynamic-driven separation mechanism. On the other hand, the interpenetrated SIFSIX-2-Cu-i allows a complete exclusion of $\mathrm{SF}_{6}$ via a molecular sieving effect with only $\mathrm{N}_{2}$ entering the pores leading to an infinite $\mathrm{N}_{2} / \mathrm{SF}_{6}$ selectivity.

The GCMC simulations allowed us to shed light in the co-adsorption mechanism at the microscopic level. The preferential adsorption of $\mathrm{SF}_{6}$ over $\mathrm{N}_{2}$ in the SIFSIX-2-Cu 
MOF was confirmed by the shape and intensities of the calculated guest-pore atomatom radial distribution functions (RDF). Some representative RDF, corresponding to the thermodynamically adsorbed fluids (equimolar bulk mixture composition) at 1 bar and $303 \mathrm{~K}$ in the SIFSIX-2-Cu and SIFSIX-2-Cu-i nanopores, are presented in Figure 4. Note that these plotted data illustrate the most representative interactions observed in the system.

These RDFs clearly confirm that the different adsorption sites of SIFSIX-2-Cu, particularly the $\mathrm{C}_{1}$ and $\mathrm{C}_{4}$ atoms of the pyridine rings, preferentially interact with $\mathrm{SF}_{6}$ since the intensities of the main peaks, which are correlated with the strength of interactions, are higher than that corresponding to $\mathrm{N}_{2}$ (Figures $4 \mathrm{a}, \mathrm{b}$ ). This can be clearly observed especially in the shape of the $\mathrm{C}_{1}-\mathrm{F}$ and $\mathrm{C}_{1}-\mathrm{N}$ RDFs for which two sharp peaks are located at 3.5 and $5.5 \AA$ in the case of the $\mathrm{C}_{1}-\mathrm{F}$ RDF (Figure 4a) and a low-intensity peak is present at $4.4 \AA$ in the case of the $\mathrm{C}_{1}-\mathrm{N}$ RDF (Figure $4 \mathrm{~b}$ ). This behavior is consistent with the higher adsorption enthalpy calculated for $\mathrm{SF}_{6}$. When also comparing the RDFs for the $\mathrm{N}_{2} / \mathrm{MOF}$ pair (Figures $4 \mathrm{~b}$, c), it can be clearly seen that the peaks become sharper and with a higher intensity in the case of the interpenetrated framework, exhibiting the strongest interaction with the $\mathrm{C}_{1}$ atoms of the pyridine rings but also more pronounced interactions with the fluorine atoms, as it can be seen in Figure 4c. In the case of SIFSIX-2-Cu-i, the F-N RDF exhibits a sharp peak located at $3.4 \AA$, whereas in the case of SIFSIX-2-Cu (Figure $4 \mathrm{~b}$ ) the corresponding RDF exhibits a relatively low-intensity peak. This increase of the peak intensity of all the RDFs can be interpreted in terms of the higher confinement degree felt by $\mathrm{N}_{2}$ in the small pore diameter of SIFSIX-2-Cu-i, which is also consistent with the significant increase of the adsorption enthalpy of $\mathrm{N}_{2}$ at low coverage $(-24.5$ $\mathrm{kJ} / \mathrm{mol})$ as compared to the value obtained in the non-interpenetrated phase $(-9.2$ $\mathrm{kJ} / \mathrm{mol}$ ). Analysis of these data explains the higher $\mathrm{N}_{2}$ uptake in the interpenetrated SIFSIX-2-Cu-i MOF, in comparison with SIFSIX-2-Cu, for all investigated bulk fluid compositions, as depicted in Figure 2.

Representative snapshots of the GCMC simulations, depicting the preferential adsorption of $\mathrm{SF}_{6}$ in the SIFSIX-2-Cu material and the exclusion of $\mathrm{SF}_{6}$ molecules via molecular sieving in the case of the interpenetrated SIFSIX-2-Cu-i framework are presented in Figure 5 for the case of the equimolar bulk mixture. As it can be clearly observed in Figure 5a, the large pores of SIFSIX-2-Cu allow the adsorption of both 
$\mathrm{SF}_{6}$ and $\mathrm{N}_{2}$ molecules and the thermodynamic-driven separation mechanism determines the preferential adsorption of $\mathrm{SF}_{6}$. Figure $5 \mathrm{~b}$ illustrates the preferential interaction between $\mathrm{SF}_{6}$ and the carbon atoms of the organic linker with characteristic host/guest distances of about $3 \AA$ consistent with the corresponding RDFs reported in Figure 4. On the other hand, as it can be clearly observed from Figure 5c, the small pore size of SIFSIX-2-Cu-i causes the complete exclusion of the larger size $\mathrm{SF}_{6}$ molecules, allowing only the adsorption of $\mathrm{N}_{2}$.

Molecular Dynamics simulations were further performed in the canonical NVT ensemble, using the calculated $\mathrm{SF}_{6}$ and $\mathrm{N}_{2}$ uptakes corresponding to 1 bar and an equimolar bulk mixture composition, to explore the diffusivity of the guest molecules in both SIFSIX-2-Cu and SIFSIX-2-Cu-i. The self-diffusion coefficients of $\mathrm{SF}_{6}$ and $\mathrm{N}_{2}$ were calculated using the well-known Einstein relation applied to the mean-square displacements (MSDs) for both guests averaged over all the MD trajectories and using a multi-time step origin. The corresponding MSDs are presented in Figure 6. In the case of SIFSIX-2-Cu, this led to $D s\left(\mathrm{SF}_{6}\right)$ and $D s\left(\mathrm{~N}_{2}\right)$ values of $2.50 \cdot 10^{-9}$ and $14.90 \cdot 10^{-9} \mathrm{~m}^{2} / \mathrm{s}$, respectively, resulting in a self-diffusivity ratio of $\sim 6$. This finding indicates that SIFSIX-2-Cu equally exhibits a satisfactory kinetic selectivity. Interestingly, the self-diffusivity for $\mathrm{N}_{2}$ in the interpenetrated SIFSIX-2-Cu-i framework drops down to the value of $2.59 \cdot 10^{-9} \mathrm{~m}^{2} / \mathrm{s}$. These differences are clearly reflected on the calculated MSDs (Figure 6). The combination of the decrease of the pore dimensions of SIFSIX-2-Cu-i with the strengthening of the fluid-pore interactions, can be considered as the main reason for this significant decrease of the self-diffusion coefficient for $\mathrm{N}_{2}$.

\section{Conclusions}

In summary, the present study revealed the predominant effect of the interpenetration of the MOF network on the separation of the $\mathrm{SF}_{6} / \mathrm{N}_{2}$ mixture. A high thermodynamic and kinetic adsorption selectivity for $\mathrm{SF}_{6}$ over $\mathrm{N}_{2}$ has been observed in the case of SIFSIX-2-Cu, whereas the steric hindrance due to the much smaller pore dimensions of the interpenetrated SIFSIX-2-Cu-i framework induces a molecular sieving effect, allowing only the adsorption of $\mathrm{N}_{2}$. This computational exploration paves the way towards the identification of adsorbents highly selective for this strategic gas mixture 
that has been only rarely explored so far using a physisorption-based separation process.

\section{Acknowledgements}

The research leading to these results has received funding from the King Abdullah University of Science and Technology (KAUST) under Center Partnership Fund Program (CPF 2910). G.M. thanks Institut Universitaire de France for its support.

\section{References}

1) L. G. Christoforou, R. J. Van Brunt, $\mathrm{SF}_{6} / \mathrm{N}_{2}$ mixtures: basic and $\mathrm{HV}$ insulation properties, IEEE Trans. Dielectr. Electr. Insul. 2 (1995), 952-1003.

2) M. Maiss, C.A.M. Brenninkmeijer, Atmospheric $\mathrm{SF}_{6}$ : Trends, sources and prospects, Environ. Sci. Technol. 32 (1998), 3077-3086.

3) X. Fang, X. Hu, G. Janssens-Maenhout, J. Wu, J. Han, S. Su, J. Zhang, J. Hu, Sulfur Hexafluoride ( $\mathrm{SF}_{6}$ ) Emission Estimates for China: An Inventory for 1990-2010 and a Projection to 2020, Environ. Sci. Technol., 47 (2013), 3848-3855.

4) S. Builes, T. Roussel, L.F. Vega, Optimization of the separation of sulfur hexafluoride and nitrogen by selective adsorption using monte carlo simulations, AIChE, 57 (2011), 962-974.

5) I. Matito-Martos, J. Álvarez-Ossorio, J. J. Gutiérez-Sevillano, M. Doblaré, A. Martin-Calvo, S. Calero, Zeolites for the selective adsorption of sulfur hexafluoride, Phys. Chem. Chem. Phys., 17 (2015), 18121-18130.

6) I. Senkovska, E. Barea, J. A. Rodríguez Navarro, S. Kaskel, Adsorptive capturing and storing greenhouse gases such as sulfur hexafluoride and carbon tetrafluoride using metal-organic frameworks, Microporous Mesoporous Mater., 156 (2012), 115120. 
7) I. Cha, S. Lee, J. D. Lee, G.-W. Lee, Y. Seo, Separation of $\mathrm{SF}_{6}$ from Gas Mixtures Using Gas Hydrate Formation, Environ. Sci. Technol., 44 (2010), 6117-6122.

8) O. Yamamoto, T. Takuma, M. Kinouchi, Recovery of $\mathrm{SF}_{6}$ from $\mathrm{N}_{2} / \mathrm{SF}_{6}$ gas mixtures by using a polymer membrane, IEEE Electr. Insul. Mag., 18 (2002), 32-37.

9) K. Inami, Y. Maeda, Y. Habuchi, M. Yoshimura, S. Hamano, H. Hama, Problems of the application of $\mathrm{N}_{2} / \mathrm{SF}_{6}$ mixtures to gas-insulated bus, Electr. Eng. Jpn., 137 (2001), 25-31.

10) H. Murase, T. Imai, T. Inohara, M. Toyoda, Use of Zeolite Filter in Portable Equipment for Recovering $\mathrm{SF}_{6}$ in $\mathrm{SF}_{6} / \mathrm{N}_{2}$ Mixtures, IEEE Trans. Electr. Insul., 11 (2004), 166-173.

11) M.B. Kim, S.J. Lee, C. Y. Lee, Y. S. Bae, High $\mathrm{SF}_{6}$ selectivities and capacities in isostructural metal-organic frameworks with proper pore sizes and highly dense unsaturated metal sites, Micropor. Mesopor. Mat., 190 (2014), 356-361.

12) P.-J. Kim, Y.-W. You, H. Park, J.-S. Chang, Y.-S. Bae, C.-H. Lee, J.-K. Suh, Separation of $\mathrm{SF}_{6}$ from $\mathrm{SF}_{6} / \mathrm{N}_{2}$ mixture using metal-organic framework MIL-100(Fe) granule, Chem. Eng. J., 262 (2015), 683-690.

13) A. Takase, H. Kanoh, T. Ohba, Wide Carbon Nanopores as Efficient Sites for the Separation of $\mathrm{SF}_{6}$ from $\mathrm{N}_{2}$, Sci. Rep., 5 (2015), 11994.

14) M.B. Kim, T.-U. Yoon, D.-Y. Hong, S.-Y. Kim, S.-J. Lee, S.-I. Kim, S.-K. Lee, J.-S. Chang, Y.B. Bae, High $\mathrm{SF}_{6} / \mathrm{N}_{2}$ selectivity in a hydrothermally stable zirconiumbased metal-organic framework, Chem. Eng. J., 276 (2015), 315-321.

15) T. Hasell, M. Miklitz, A. Stephenson, M. A. Little, S.Y. Chong, R. Clowes, L. Chen, D. Holden, G.A. Tribello, K.E. Jelfs, A.I. Cooper, Porous Organic Cages for Sulfur Hexafluoride Separation, J. Am. Chem. Soc., 138 (2016), 1653-1659.

16) I. Skarmoutsos, G. Tamiolakis, G.E. Froudakis, Highly selective separation and adsorption-induced phase transition of $\mathrm{SF}_{6}-\mathrm{N}_{2}$ fluid mixtures in three-dimensional carbon nanotube networks, J. Supercrit. Fluids, 113 (2016), 89-95.

17) C.Y. Chuah, K. Goh, T.-H. Bae, Hierarchically structured HKUST-1 nanocrystals for enhanced $\mathrm{SF}_{6}$ capture and recovery, J. Phys. Chem. C, 121 (2017), 6748-6755. 
18) K. Shiojiri, Y. Yanagisawa, A. Yamasaki, F. Kiyono, Separation of F-gases (HFC-134a and $\mathrm{SF}_{6}$ ) from gaseous mixtures with nitrogen by surface diffusion through a porous Vycor glass membrane, J. Membr. Sci., 282 (2006), 442-449.

19) L. Shao, B. T. Low, T. S. Chung, A. R. Greenberg, Polymeric membranes for the hydrogen economy: Contemporary approaches and prospects for the future, J. Membr. Sci., 327 (2009), 18-31.

20) J.-W. Choi, S. Lee, B. An, S.-B. Kim, S.-H. Lee, Separation of sulfur hexafluoride from a nitrogen/sulfur hexafluoride mixture using a polymer hollow fiber membrane, Water Air Soil Pollut., 225 (2014), 1807.

21) A. Wolińska-Grabczyk, A. Jankowski, R. Sekuła, B. Kruczek, Separation of SF6 from Binary Mixtures with $\mathrm{N}_{2}$ Using Commercial Poly(4-Methyl-1-Pentene) Films, Sep. Sci. Technol., 46 (2011), 1231-1240.

22) Themed collection Metal Organic Frameworks, Chem. Soc. Rev., 46 (2017), 3104.

23) K. Adil, Y. Belmabkhout, R. S. Pilai, A. Cadiau, P. M. Bhatt, A. H. Assen, G. Maurin, M. Eddaoudi, Gas/vapour separation using ultra-microporous metal-organic frameworks: insights into the structure/separation relationship, Chem. Soc. Rev., 46 (2017), 3402.

24) Y.-N. Gong, D.-C. Zhong, T.-B. Lu, Interpenetrating metal-organic frameworks, CrystEngComm, 18 (2016), 2596-2606.

25) H.-L. Jiang, T.A. Makal, H.-C. Zhou, Interpenetration control in metal-organic frameworks for functional applications, Coord. Chem. Rev., 257 (2013), 2232-2249.

26) P. Nugent, Y. Belmabkhout, S. D. Burd, A.J. Cairns, R. Luebke, K. Forrest, T. Pham, S. Ma, B. Space, L. Wojtas, M. Eddaoudi and M.J. Zaworotko, Porous materials with optimal adsorption thermodynamics and kinetics for $\mathrm{CO}_{2}$ separation, Nature, 495 (2013), 80-84.

27) I. Skarmoutsos, Y. Belmabkhout, K. Adil, M. Eddaoudi, G. Maurin, $\mathrm{CO}_{2}$ Capture Using the SIFSIX-2-Cu-i Metal-Organic Framework: A Computational Approach, J. Phys. Chem. C, 121 (2017), 27462-27472. 
28) D. Dellis, J. Samios, Molecular force field investigation for Sulfur Hexafluoride: A computer simulation study, Fluid Phase Equil., 291 (2010), 81-89.

29) C. Murthy, K. Singer, M. Klein, I. R. McDonald, Pairwise additive effective potentials for nitrogen, Mol. Phys., 41 (1980), 1387-1399.

30) I. Skarmoutsos, M. Eddaoudi, G. Maurin, Peculiar Molecular Shape and Size Dependence of the Dynamics of Fluids Confined in a Small-Pore Metal-Organic Framework, J. Phys. Chem. Lett., 9 (2018), 3014-3020.

31) S.L. Mayo, B. D. Olafson, W.A. Goddard III, DREIDING: a generic force field for molecular simulations, J. Phys. Chem., 94 (1990), 8897-8909.

32) A.K. Rappe, C.J. Casewit, K.S. Colwell, W.A. Goddard III, W.M. Skiff, UFF, a full periodic table force field for molecular mechanics and molecular dynamics simulations, J. Am. Chem. Soc., 114 (1992), 10024-10035.

33) J.P. Perdew, K. Burke, M. Ernzerhof, Generalized Gradient Approximation Made Simple, Phys. Rev. Lett., 77 (1996), 3865-3868.

34) S. Grimme, J. Antony, S. Ehrlich, H. Krieg, A consistent and accurate ab initio parametrization of density functional dispersion correction (DFT-D) for the 94 elements H-Pu, J. Chem. Phys., 132 (2010), 154104.

35) J. VandeVondele, M. Krack, F. Mohamed, M. Parrinello, T. Chassaing, J. Hutter, QUICKSTEP: Fast and accurate density functional calculations using a mixed Gaussian and plane waves approach, Comput. Phys. Commun., 167 (2005), 103-128.

36) J. Hutter, M. Ianuzzi, F. Schiffmann, J. VandeVondele, cp2k: atomistic simulations of condensed matter systems, WIREs Comput. Mol. Sci., 4 (2014), 15-25.

37) J. VandeVondele, J. Hutter, Gaussian basis sets for accurate calculations on molecular systems in gas and condensed phases, J. Chem. Phys., 127 (2007), 114105.

38) S. Goedecker, M. Teter, J. Hutter, Separable dual-space Gaussian pseudopotentials, Phys. Rev. B, 54 (1996), 1703-1710. 
39) Q. Yang, C. Zhong, Molecular Simulation of Carbon Dioxide/Methane/Hydrogen Mixture Adsorption in Metal-Organic Frameworks, J. Phys. Chem. B, 110 (2006), 17776-17783.

40) D.-Y. Peng, D.B. Robinson, A New Two-Constant Equation of State, Ind. Eng. Chem. Fundamen., 15 (1976), 59-64.

41) M. P. Allen, D. J. Tildesley, Computer Simulations of Liquids, Oxford University Press, Oxford, 1987.

42) W. G. Hoover, Canonical dynamics: Equilibrium phase-space distributions, Phys. Rev. A, 31 (1985), 1695-1697.

43) W. Smith, T. R. Forester, DL_POLY_2.0: a general-purpose parallel molecular dynamics simulation package, J. Mol. Graphics, 14 (1996), 136-141.

44) R. Sun, C.-W. Tai, M. Strømme, O. Cheung, Hierarchical porous carbon synthesized from novel porous amorphous calcium or magnesium citrate with enhanced $\mathrm{SF}_{6}$ uptake and $\mathrm{SF}_{6} / \mathrm{N}_{2}$ selectivity, ACS Appl. Nano Mater., DOI: 10.1021/acsanm.8b02005 (2019). 


\section{TABLES}

Table 1: Partial charges and Lennard-Jones parameters corresponding to each type of atoms in the SIFSIX-2-Cu-i and SIFSIX-2-Cu supercells. For the terminal nitrogen and hydrogen atoms the symbols NT and HT have been used, respectively. The charges of the terminal nitrogen atoms NT of the SIFSIX-2-Cu supercell have been slightly adjusted to give a total zero charge to the supercell. The different carbon types $\mathrm{C}_{1}-\mathrm{C}_{4}$ are depicted in Figure 1 in the manuscript.

\begin{tabular}{|c|c|c|c|}
\hline Interaction Site & $\mathbf{q}(|\mathbf{e}|)$ & $\boldsymbol{\sigma}(\mathbf{\AA})$ & $\boldsymbol{\varepsilon}(\mathbf{K})$ \\
\hline $\mathbf{C u}$ & 0.700 & 3.2950 & 2.5178 \\
\hline $\mathbf{S i}$ & 0.734 & 3.8264 & 202.2900 \\
\hline $\mathbf{F}$ & -0.370 & 3.0930 & 36.4830 \\
\hline $\mathbf{N}$ & -0.182 & 3.6621 & 38.9750 \\
\hline $\mathbf{N T}$ & -0.69635 & 3.6621 & 38.9750 \\
\hline SIFSIX-2-Cu-i) & & & 38.9750 \\
\hline $\mathbf{N T}$ & -0.561875 & 3.6621 & 7.6490 \\
\hline $\mathbf{S I F S I X - 2 - C u})$ & & & 7.6490 \\
\hline $\mathbf{H}$ & 0.136 & 2.8464 & 47.8570 \\
\hline $\mathbf{H T}$ & 0.276 & 2.8464 & 47.8570 \\
\hline $\mathbf{C}_{\mathbf{1}}$ & -0.226 & 3.4730 & 47.8570 \\
\hline $\mathbf{C}_{\mathbf{2}}$ & 0.088 & 3.4730 & 47.8570 \\
\hline $\mathbf{C}_{\mathbf{3}}$ & -0.140 & 3.4730 & 3.4730 \\
\hline $\mathbf{C}_{\mathbf{4}}$ & 0.300 & & \\
\hline
\end{tabular}


Table 2: Partial charges and Lennard-Jones parameters corresponding to each type of interaction sites in the $\mathrm{SF}_{6}$ molecule.

\begin{tabular}{|c|c|c|c|}
\hline Interaction Site & $\mathbf{q}(|\mathbf{e}|)$ & $\boldsymbol{\sigma}(\AA)$ & $\boldsymbol{\varepsilon}(\mathbf{K})$ \\
\hline $\mathbf{S}$ & 0.66 & 3.228 & 165.14 \\
\hline $\mathbf{F}$ & -0.11 & 2.947 & 27.02 \\
\hline
\end{tabular}

S-F Bond Length: $1.564 \AA$

Table 3: Partial charges and Lennard-Jones parameters corresponding to each type of interaction sites in the $\mathrm{N}_{2}$ molecule.

\begin{tabular}{|c|c|c|c|}
\hline Interaction Site & $\mathbf{q}(|\mathbf{e}|)$ & $\boldsymbol{\sigma}(\stackrel{\AA}{)})$ & $\boldsymbol{\varepsilon}(\mathbf{K})$ \\
\hline $\mathbf{N}$ & -0.482 & 3.32 & 36.4 \\
\hline $\begin{array}{c}\text { C.O.M. } \\
\text { (center of mass) }\end{array}$ & 0.964 & - & - \\
\hline
\end{tabular}

N-N Bond Length: $1.098 \AA$ 
FIGURES

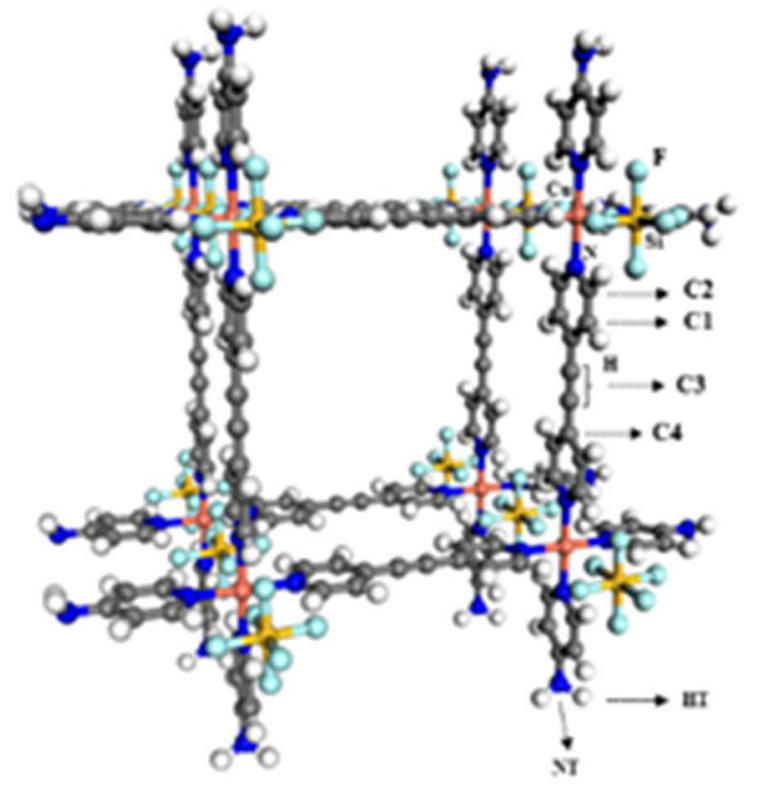

Figure 1 

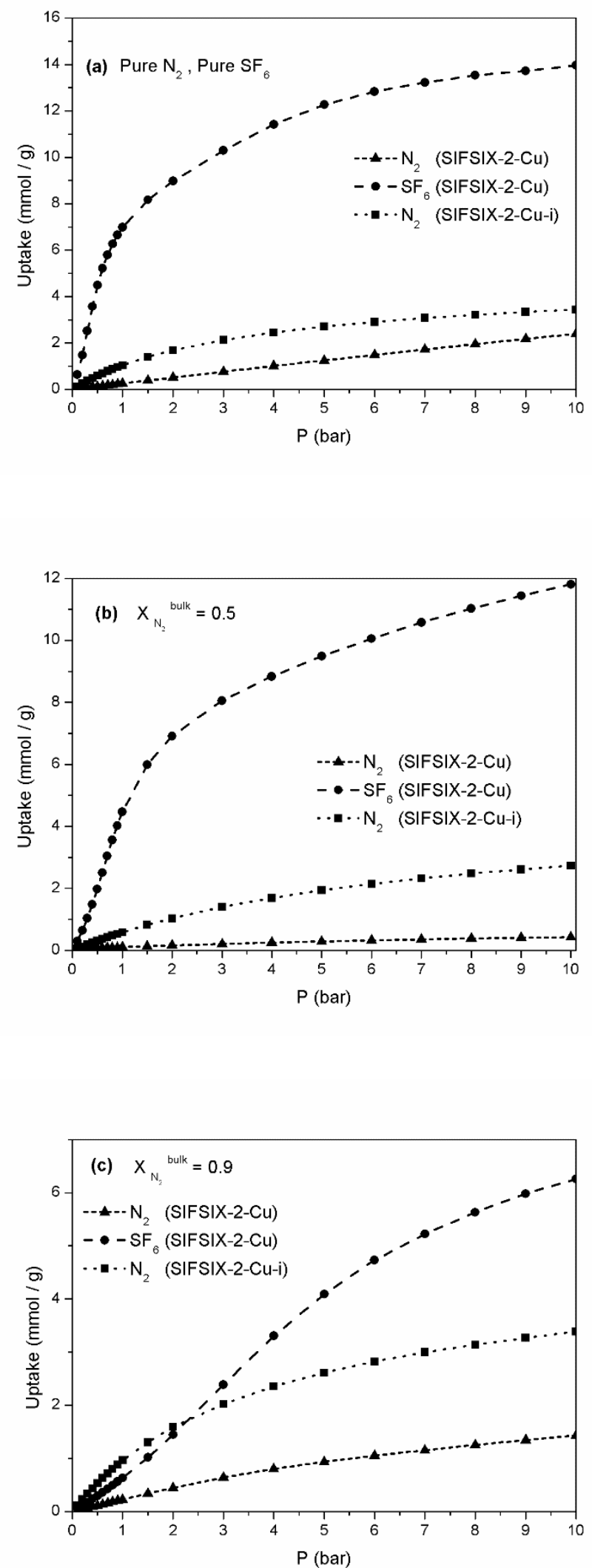

Figure 2 


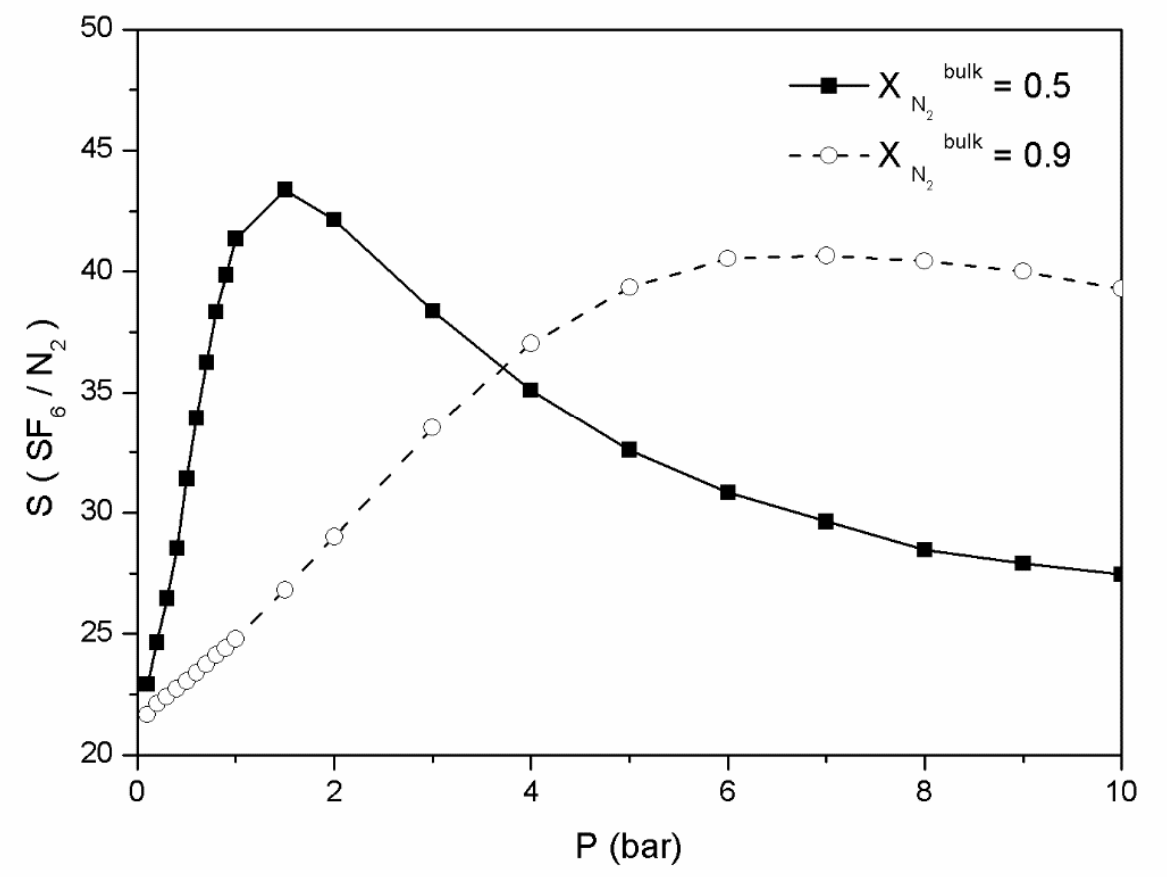

Figure 3 

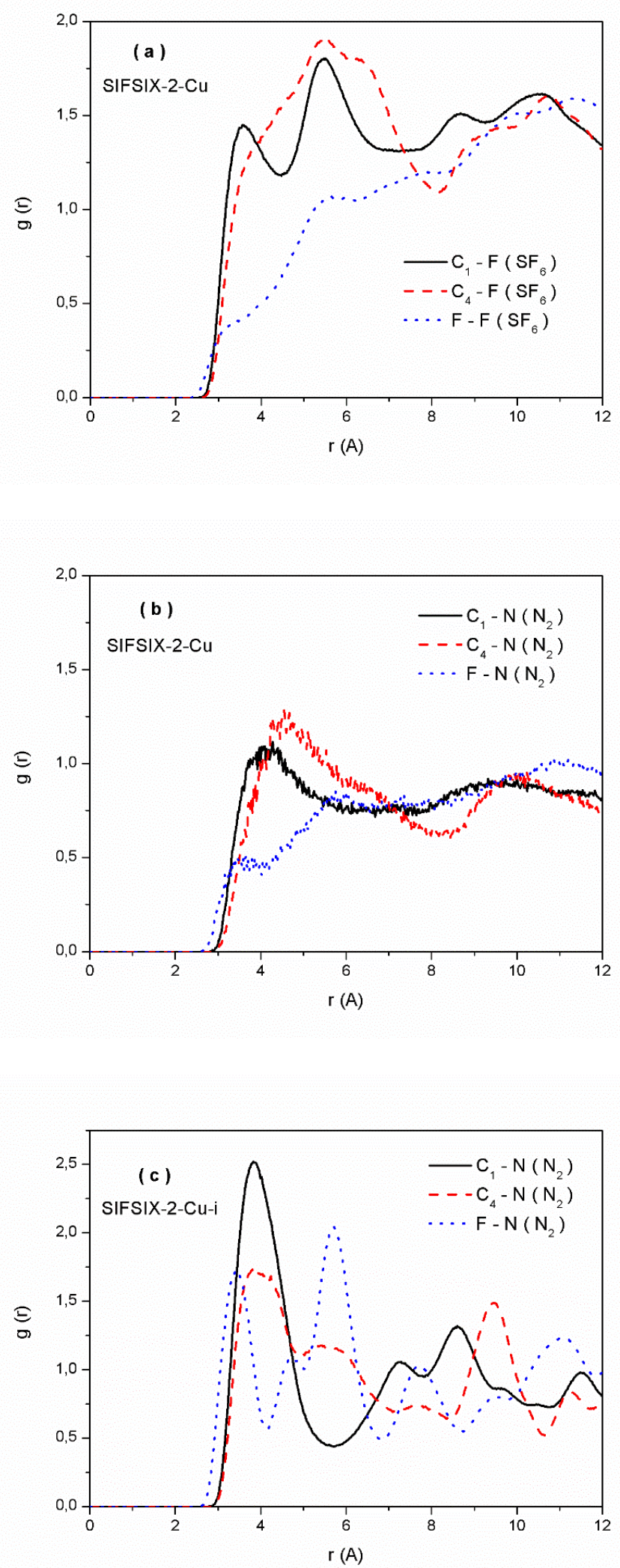

Figure 4 
(a)

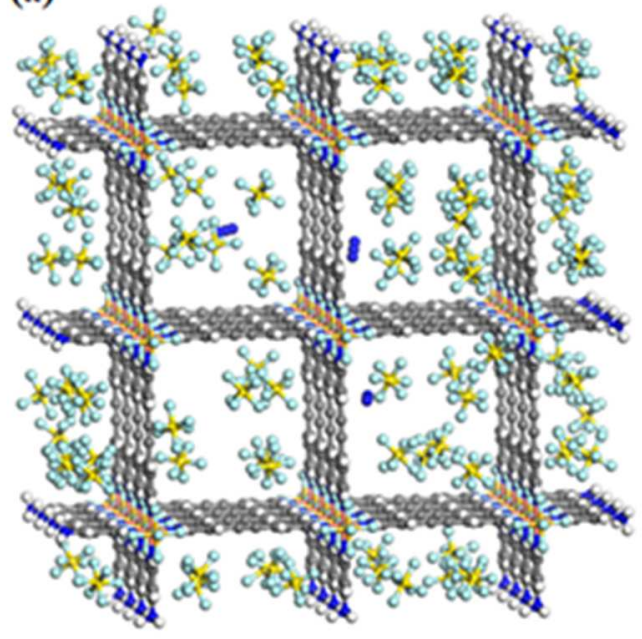

(b)
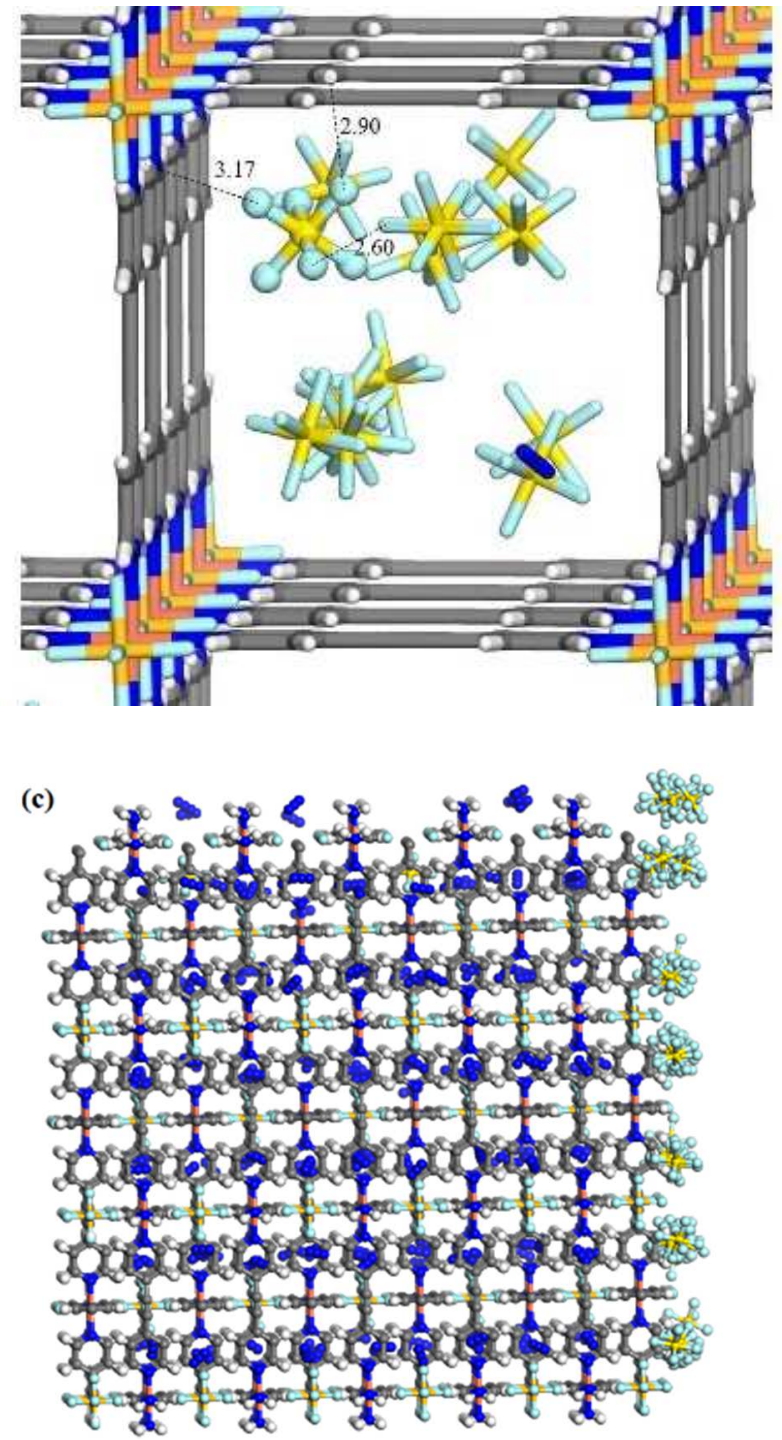

Figure 5 


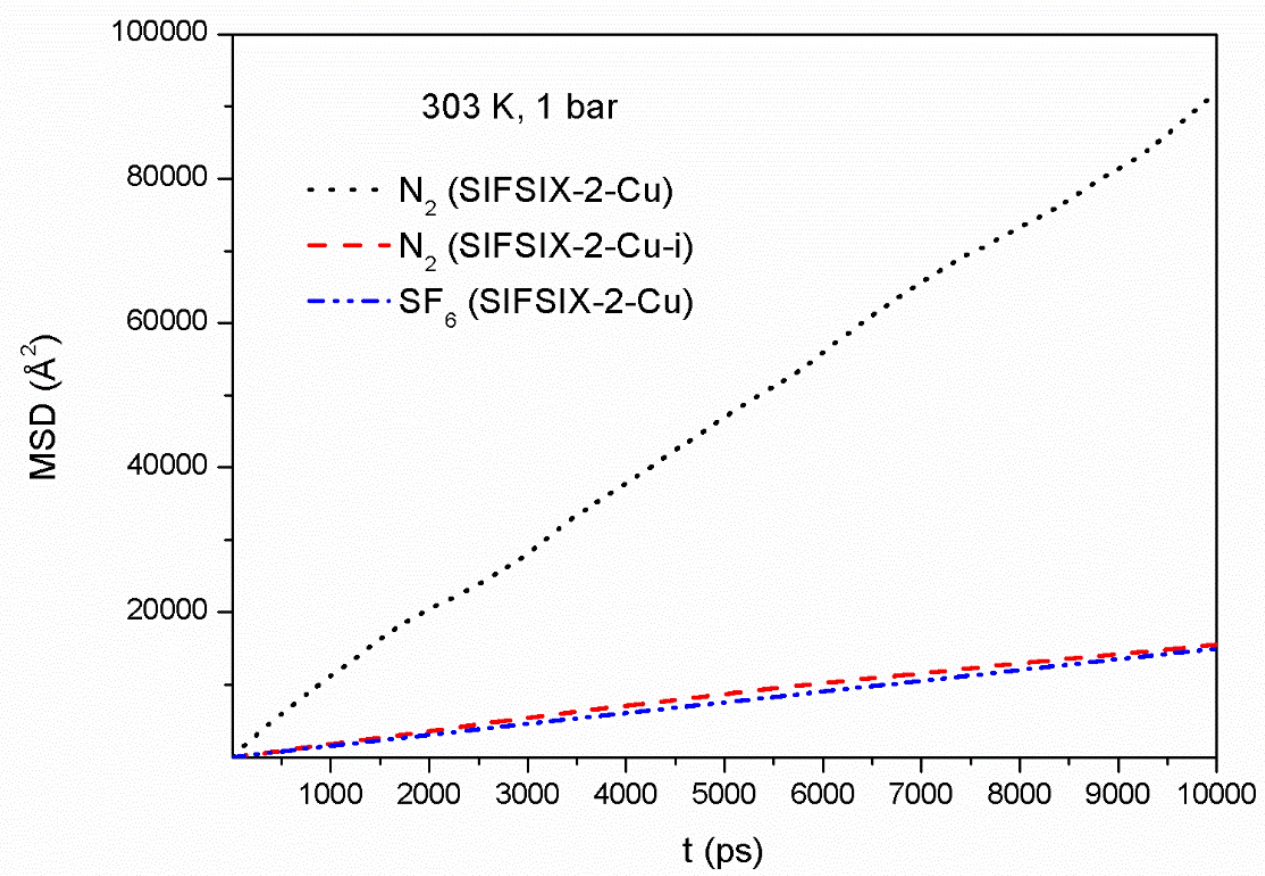

Figure 6 


\section{FIGURE CAPTIONS}

Figure 1: Names of the interaction sites corresponding to a model building block of the SIFSIX-2-Cu framework.

Figure 2: The binary mixture adsorption isotherms for $\mathrm{SF}_{6}$ and $\mathrm{N}_{2}$ corresponding to (a) the pure fluids, (b) the equimolar (1:1) and (c) the 1:9 $\mathrm{SF}_{6} / \mathrm{N}_{2}$ bulk mixtures at 303 $\mathrm{K}$ for SIFSIX-2-Cu and SIFSIX-2-Cu-i.

Figure 3: The calculated thermodynamic adsorption selectivity for $\mathrm{SF}_{6}$ over $\mathrm{N}_{2}$, corresponding to the bulk (1:1) and (1:9) $\mathrm{SF}_{6} / \mathrm{N}_{2}$ binary mixtures, in the case of SIFSIX-2-Cu as a function of pressure.

Figure 4: Representative interatomic guest-pore radial distribution functions, corresponding to the thermodynamically adsorbed components of an equimolar bulk mixture at 1 bar and $303 \mathrm{~K}$, in SIFSIX-2-Cu and SIFSIX-2-Cu-i.

Figure 5: Representative snapshots of the GCMC simulations corresponding to an equimolar bulk mixture, depicting the preferential adsorption of $\mathrm{SF}_{6}$ in SIFSIX-2-Cu ( $a$ and $b$ ) and the molecular sieving effect, allowing only the adsorption of $\mathrm{N} 2$ in the case of SIFSIX-2-Cu-i (c).

Figure 6: Mean-square displacements for the adsorbed $\mathrm{N}_{2}$ and $\mathrm{SF}_{6}$ molecules in the two materials, corresponding to an equimolar bulk mixture at $303 \mathrm{~K}$ and 1 bar. This graph does not report the plots for $\mathrm{SF}_{6}$ in SIFSIX-2-Cu-i since this molecule cannot enter the pore of this MOF. 